\title{
Rotational Speed Control of Multirotor UAV's Propulsion Unit based on Fractional-order PI Controller
}

\author{
Wojciech Giernacki*, Talar Sadalla*, Jarosław Gośliński*, Piotr Kozierski**,*, João P. Coelho***, Saša Sladić**** \\ Poznan University of Technology, Piotrowo 3a Street, 60-965 Poznan, Poland*,** \\ *Faculty of Electrical Engineering, Institute of Control and Information Engineering \\ ** Faculty of Computing, Chair of Control and Systems Engineering, Division of Signal Processing and Electronic Systems \\ *** Instituto Politécnico de Bragança, Campus de Santa Apolónia, Apartado 1134, 5301-857 Bragança, Portugal \\ **** Faculty of Engineering, University of Rijeka, Vukovarska 58, 51000 Rijeka, Croatia \\ E-mail: wojciech.giernacki@put.poznan.pl
}

\begin{abstract}
In this paper the synthesis of a rotational speed closed-loop control system based on a fractional-order proportional-integral (FOPI) controller is presented. In particular, it is proposed the use of the SCOMR-FOPI procedure as the controller tuning method for an unmanned aerial vehicle's propulsion unit. In this framework, both the Hermite-Biehler and Pontryagin theorems are used to predefine a stability region for the controller. Several simulations were conducted in order to try to answer the questions - is the FOPI controller good enough to be an alternative to more complex FOPID controllers? In what circumstances can it be advantageous over the ubiquitous PID? How robust this fractional-order controller is regarding the parametric uncertainty of considered propulsion unit model?
\end{abstract}

Keywords - fractional-order controller; speed controller; FOPI controller; linear BLDC motor model; propulsion unit; motor-rotor model; Hermite-Biehler and Pontryagin theorems

\section{INTRODUCTION}

There are two main objectives when designing a controller system: to ensure the control system stability and subsequently to provide the highest possible control precision in the presence of disturbances and uncertainties. Those subjects have been addressed by many researchers working on unmanned aerial vehicles (UAVs) - especially within several contexts such as the control algorithms [1],[9], optimization of the UAV's construction [3] or ensuring fault-tolerant control [20]. Many of those solutions are inspired on ideas used for fixed-wing UAVs [7],[27].

Bearing in mind the cascade control structure used in UAVs, it is impossible to think about mission planning [18] or flights autonomy [6],[21] without ensuring appropriate solutions in the lower layers of a control architecture (position and orientation control). At this bottom level one can find the system known as Electronic Speed Control (ESC). This module is responsible to drive the DC motor via appropriately fast changes of Pulse Width Modulation (PWM) duty cycles, leading to the generated thrust and torque. Therefore, it is important to use appropriate motor-rotor units [28], with the proper sizes [5], proper arrangements [2] and appropriate controller type. It should be noted that this is the fastest control loop in the architecture of multirotor UAVs, which leads to the highest time requirements and saturations to be met.

\section{PROBLEM STATEMENT}

The rotational speed control is a deeply investigated problem, but it is still an open area regarding the application of modern techniques based on control theory. For UAVs there are a myriad of distinct control systems approaches, some more complex and others simpler but with higher efficiency. In the literature a bigger attention is given to this second type due to several reasons such as its potential applicability in the lowest layer of rotational speed control and simplicity regarding its mathematical formulation. Regarding this concept and the currently used solutions, the main idea aroses: why not to extend the high flexibility and simplicity of ProportionalIntegral-Derivative (PID) control to fractional-order's counterpart to improve the control quality by using its leading advantage. Namely the possibility of more accurate shape fitting of closed loop system's frequency characteristics, not restricted only to $20 \log$ (gain). This can be attained by the introduction of fractional-order in the notation of integrator and differentiator polynomials parts of controller. Thus, one obtains two additional design parameters (orders of the integrator and differentiator: $\lambda$ and $\mu$ ). Their choice depends on the shape of time and frequency characteristics of the closed-loop control system [13].

In the paper [14], the author proposed a controller in the $\mathrm{PI}^{\lambda} \mathrm{D}^{\mu}$ (FOPID - Fractional-Order PID) structure for the problem described in Sections 2-3. The main difficulty (but respectively, as it is shown, also the possibility) in the proposed solution is the controller tuning with five separate degrees of freedom (controller sets). According to the defined cost function by using particle swarm optimization algorithm (see [13]), it is possible to ensure the tracking of reference signal (rotational speed of motor-rotor unit model used in UAVs) in a more efficient way than for the PID and CDM (Coefficient Diagram Method) controllers. Results are presented in [11]. 
The two main problems observed in the previous solutions were the need to tune the five controller parameters and the lack of clear proposals for methods of the stability assurance, analysis and assessment in the procedure for the control system synthesis with the proposed controller type. In order to meet these problems, in the present work, a simpler controller structure is used: $\mathrm{PI}^{\lambda}$ (or alternatively named FOPI), to verify if by using its advantages, it is possible to propose an efficient controller, with better performance than PID and FOPID. The second issue is to answer to the question: how robust is this controller on parametric uncertainty? Such a comparative analysis and research are proposed for the first time and are the novelty and contribution of this paper.

The paper is organized as follows - in Section III, SCoMRFOPI procedure is defined and explained. A synthetic information about propulsion unit model proposed for tests is provided in Section IV. In Sections V and VI, theory about fractional-order controllers and Hermite-Biehler and Pontryagin Theorems, is presented. Section VII describes FOPI controller synthesis and the system stability analysis. Last two sections provide information about conducted tests and conclusion.

\section{SCOMR-FOPI PROCEDURE}

The most general form of the procedure/algorithm for modeling motor-rotor units and for the closed-loop system synthesis, here named SCoMR-FOPI (Modeling and Rotational Speed Control of Motor-Rotor Unit) with the FOPI type controller, assumes the form:

1. Record the possible time characteristics of motor-rotor unit for various types of reference/input signals.

2. By using parametric estimation methods (i.e. Küpfmüller, Strejc or graphical method) define/calculate the parameters values for the predefined plant model structure (i.e. transfer function structure) and perform the model validation on other recorded data sets from test bed.

3. For the controller in the FOPI structure described by the transfer function, use the Hermite-Biehler and Pontryagin theorems to find the controller gains of $K_{P}, K_{I}$ and degree of integrator part's polynomial $\lambda$, for which the closed-loop control system is stable.

4. For the designated search space $\left(K_{P}, K_{I}\right.$ and $\left.\lambda\right)$ use the chosen algorithm to find the optimum value of the cost function (for example: minimum of the Integral of Absolute Error - IAE or minimum of the Integral of Squared Error ISE).

5. Generate time courses for some of best results (those with small values of cost function).

6. Select the solution ( $K_{P}, K_{I}$ and $\lambda$ set value) for which the time courses meet the expectations (for example: one that provides small/no overshoot, short lag/settling time, small/no control error, etc.).

NOTE: Because the controller set search is based on integral quality indicators and they do not take directly into account the shape of the generated time courses of closed-loop system (only describe the numerical deviation between the output signal and the reference one), there is a strong need for use the steps 5 and 6 of the SCoMR-FOPI algorithm. In the following sections, more details on each algorithm step, are presented.

\section{MOdEL OF PROPULSION UNIT}

In order to fulfil the needs of rotational speed control in a real ESC system, a special test bed was conducted at the Institute of Control and Information Engineering of the Poznan University of Technology. Based on the recorded time courses (relation between the motor angular speed and the applied voltage [11]), one can assume the existence of a motor-rotor unit mathematical model that describes well enough the device dynamics. Regarding previous studies and experiences, a number of differentiated models were proposed, subsequently conditioning the control law and the controller type, which can be used to build the control system. Examples can be found in several works. In [11] an approximation of motor-rotor unit dynamics by linear second-order inertial model was proposed. Authors of references [14-16] designed a first-order inertia model with pure time delay, which can be approximated by the second-order inertial model. In [24] the second-order inertial plant model with time delay was developed. In [12] a fuzzy model of coaxial propulsion unit (first-order inertial element with the varying time constant) was defined.

It was decided to record characteristics of the $A X I 2814 / 12$ GOLD LINE BLDC motor from T-Model Motors company with three-bladed propeller GWS-HD9050x3-SW 9x5". More details about the used test bed, dedicated DYNO Terminal software and acquisition works, are provided in [11]. Also, in [13], a comprehensive physical characteristic and justification for the choice of that particular motor-rotor unit, are provided.

From the tests performed to obtain the transfer function of the motor-rotor model (relations between angular speed $\Omega$ and applied voltage $V$ ), one decided to use a first-order inertial model with transport delay $L$ :

$$
G(s)=\frac{\Omega(s)}{V(s)}=\frac{b_{0}}{a_{1} s+a_{0}} e^{-s L},
$$

and two approximation approaches with the use of:

- IDENT library of MATLAB ${ }^{\circledR}$ software,

- ad-hoc graphical method (Küpfmüller) and arbitrary selection of the model parameters.

From validation experiments, better curve fitting and, as a result, parameters of motor-rotor unit model's transfer function, provided the second method.

\section{FOPI CONTROLLER THEORETICAL BACKGROUND}

Theoretical basics of fractional calculus are presented in [10] and [19]. Examples of fractional-order control applications in relation to position/orientation control of multirotor UAVs can be found in [26] and more examples of such controllers' synthesis in the work [10]. In the articles [22] and [23], information about time and frequency domain analysis are, respectively, provided. 
According to [10] fractional calculus is a generalization of differentiation and integration to non-integer order fundamental (continuous integro-differential) operator ${ }_{a} D_{t}^{r}$ defined as:

${ }_{a} D_{t}^{r}=\left\{\begin{array}{cl}\frac{d^{r}}{d t^{r}} & \Re(r)>0 \\ 1 & \Re(r)=0, \\ \int_{a}^{t}(d \tau)^{-r} & \Re(r)<0\end{array}\right.$

where $a$ and $t$ are operation limits and $r$ is the operation order (usually $r$ is a real value).

From the Riemann-Liouville definition of the fractional differo-integral:

${ }_{a} D_{t}^{r}(t)=\frac{1}{\Gamma(n-r)}\left(\frac{d}{d t}\right)^{n} \int_{a}^{t} \frac{f(\tau)}{(t-\tau)^{1-n+\alpha}} d \tau$

for $n-1<r<n$ where $\Gamma($.$) is the Euler's Gamma function,$ in analogy to integer-order systems, for non-integer order systems, one may use the Laplace transform of equation (3), which is defined as:

$\int_{0}^{\infty} e^{-s t}{ }_{0} D_{t}^{r} f(t) d t=s^{r} F(s)-\left.\sum_{k=0}^{n-1} s^{k}{ }_{0} D_{t}^{r-k-1} f(t)\right|_{t=0}$

for $n-1<r<n$, where $s$ denotes the Laplace transform variable and $s=j \omega$.

From the equation (4), it is hence possible to set the structure of FOPI controller type to the form of transfer function:

$C(s)=K_{P}+K_{I} s^{-\lambda}$,

where $K_{P}$ is the proportional gain, $K_{I}$ - integration gain and $\lambda$ is a positive real number.

The quasi-polynomial $\delta(s)$, which describes the closed-loop characteristic equation of the system from Fig. 1 is given by:

$\delta(s)=\left(b_{0} K_{P}+b_{0} K_{I} s^{-\lambda}\right) e^{-s L}+a_{1} s+a_{0}$.

In order to find the controller parameters sets, Hermite-Biehler and Pontryagin theorems are used.

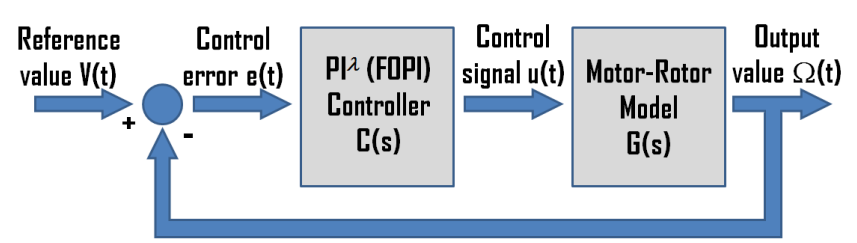

Fig. 1. Block diagram of closed-loop control system with the FOPI controller type and motor-rotor model

\section{HERMITE-BIEHLER AND PONTRYAGIN THEOREM}

Theorem 1. Hermite-Biehler Theorem ([8],[17]).

Let $\delta$ be a complex function of $\omega$ described by equation:

$\delta^{*}(j \omega)=\delta_{r}^{*}(\omega)+j \delta_{i}^{*}(\omega)$,

where $\delta_{r}^{*}(\omega)$ and $\delta_{i}^{*}(\omega)$ represent the real and imaginary parts of $\delta^{*}(j \omega)$. The $\delta^{*}(j \omega)$ is stable if:

1) $\delta_{r}^{*}(\omega)$ and $\delta_{i}^{*}(\omega)$ only have simple real roots and these are interlaced;

2) $\delta_{i}^{\prime^{*}}(\omega) \delta_{r}^{*}(\omega)-\delta_{i}^{*}(\omega) \delta_{r}^{\prime *}(\omega)>0$, for some $\omega=\omega-$ in $(-\infty,+\infty)$,

Where $\delta_{i}^{\prime *}(\omega)$ and $\delta_{r}^{\prime *}(\omega)$ are the derivatives of $\delta_{i}^{*}(\omega)$ and $\delta_{r}^{*}(\omega)$ with respect to $\omega$. An important step is to ensure that $\delta_{i}^{*}(\omega)$ and $\delta_{r}^{*}(\omega)$ only have real roots. This can be achieved by applying the Pontryagin Theorem.

Theorem 2. Pontryagin Theorem

Let $\delta^{*}(s)$ be described by the equation (7) assuming $s=j \omega$.

To assure that $\delta_{i}^{*}(\omega)=0$ and $\delta_{r}^{*}(\omega)=0$ only have real roots, it must be assured that in intervals

$-2 l \pi+\eta \leq \omega \leq 2 l \pi+\eta \quad l=1,2,3, \ldots$,

$\delta_{i}^{*}(\omega)$ and $\delta_{r}^{*}(\omega)$ have exactly $4 l N+M$ roots. For situations where characteristic equation is of fractional order, the $\delta_{i}^{*}(\omega)$ and $\delta_{r}^{*}(\omega)$ must have $4 l([N]+1)+[M]+1$ roots, where [...] represents the integer part, and $N$ and $M$ are taken as degree of the numerator and denominator polynomials of the integer part.

Proofs can be found in [8].

As it is shown in [24], it is necessary to rewrite the $\delta(s)$ quasi-polynomial as:

$\delta^{*}(s)=b_{0} K_{P} s^{\lambda}+b_{0} K_{I}+\left(a_{1} s+a_{0}\right) e^{s L} s^{\lambda}$.

Assuming that $g=L s$ and $\lambda=a / b$ :

$\delta^{*}(g)=b_{0} K_{P}(g / L)^{a / b}+b_{0} K_{I}+\left(a_{1} g / L+a_{0}\right) e^{g}(g / L)^{a / b}$.

Thus, for $g=j \omega, \delta^{*}(j \omega)$ becomes:

$\delta^{*}(j \omega)=b_{0} K_{P}(j \omega / L)^{a / b}+b_{0} K_{I}$
$+\left(a_{1} j \omega / L+a_{0}\right) e^{j \omega}(j \omega / L)^{a / b}$.

Replacing the $\mathrm{e}^{\mathrm{j} \omega}$ with $\cos (\omega)+\mathrm{j} \sin (\omega)$, the real $\delta_{r}^{*}(\omega)$, and imaginary $\delta_{i}^{*}(\omega)$, parts of $\delta *(\omega)$ can be described by: 


$$
\begin{gathered}
\delta_{r}^{*}(\omega)=\left[b_{0} K_{P}+a_{0} \cos (\omega)-\frac{a_{1} \omega \sin (\omega)}{L}\right] . \\
\cdot\left|\operatorname{Re}(j)^{a / b}\right| \frac{\left|\omega^{a / b}\right|}{L^{a / b}}+b_{0} K_{I}-\left[\frac{a_{1} \omega}{L} \cos (\omega)+a_{0} \sin (\omega)\right] . \\
\cdot\left|\operatorname{Im}(j)^{a / b}\right| \frac{\left|\omega^{a / b}\right|}{L^{a / b}} \operatorname{sign}(\omega), \\
\delta_{i}^{*}(\omega)=\left[b_{0} K_{P}+a_{0} \cos (\omega)-\frac{a_{1} \omega \sin (\omega)}{L}\right] . \\
\cdot\left|\operatorname{Im}(j)^{a / b}\right| \frac{\omega^{a / b} \mid}{L^{a / b}} \operatorname{sign}(\omega)-\left[\frac{a_{1} \omega}{L} \cos (\omega)+a_{0} \sin (\omega)\right] . \\
\cdot\left|\operatorname{Re}(j)^{a / b}\right| \frac{\omega^{a / b} \mid}{L^{a / b}} .
\end{gathered}
$$

According to the Pontryagin Theorem $\delta_{i}^{*}(\omega)=0$ and $\delta_{r}^{*}(\omega)=0$. The parameter $K_{P}$ can be described by:

$$
\begin{gathered}
K_{P}=\left[-\frac{a_{0}}{b_{0}} \cos (\omega)+\frac{a_{1} \omega}{b_{0} L} \sin (\omega)\right]+ \\
-\left[\frac{a_{1} \omega}{b_{0} L} \cos (\omega)+\frac{a_{0}}{b_{0}} \sin (\omega)\right] \frac{\operatorname{Re}(j)^{a / b} \mid}{\left|\operatorname{Im}(j)^{a / b}\right|} \operatorname{sign}(\omega) .
\end{gathered}
$$

One can rewrite the $\delta_{r}^{*}(\omega)$ as:

$\delta_{r}^{*}(\omega)=b_{0} K_{I}-m(\omega) K_{P}-b(\omega)$

$m(\omega)=-\left|\operatorname{Re}(j)^{a / b}\right| \frac{\left|\omega^{a / b}\right|}{L^{a / b}}$,

$b(\omega)=\left[-a_{0} \cos (\omega)+\frac{a_{1} \omega}{L} \sin (\omega)\right]\left|\operatorname{Re}(j)^{a / b}\right| \frac{\left|\omega^{a / b}\right|}{L^{a / b}}$

$-\left[a_{0} \sin (\omega)+\frac{a_{1} \omega}{L} \cos (\omega)\right] \operatorname{Im}(j)^{a / b} \mid \frac{\left|\omega^{a / b}\right|}{L^{a / b}} \operatorname{sign}(\omega)$.

In accordance with [4], the range of parameters $K_{I}$ that assures closed-loop stability must met the conditions:

$$
\begin{gathered}
\max \left(-m_{j}(\omega) K_{P}-b_{j}(\omega)\right)<K_{I}, \\
K_{I}<\min \left(-m_{j}(\omega) K_{P}-b_{j}(\omega)\right) \quad j=0,1,2, \ldots
\end{gathered} .
$$

Since $\delta_{i}^{*}(\omega)$ is an odd function, it has the root at $\omega=0$. Thus, for $\omega=\omega_{0}=0$ :

$$
\delta_{i}^{*}(\omega)=b_{0} K_{P}+a_{0} .
$$

To ensure the interlace property between $\delta_{r}^{*}(\omega)$ and $\delta_{i}^{*}(\omega)$ one must impose:

$$
\delta_{i}^{*}(\omega)>0 \Rightarrow b_{0} K_{P}+a_{0}>0 \Rightarrow K_{P}>-a_{0} / b_{0} .
$$

\section{CONTROLLER SYNTHESIS AND SYSTEM STABILITY ANALYSIS}

In order to determine the permissible space of controller sets $\left(K_{P}, K_{I}\right.$ and $\left.\lambda\right)$ for which the closed-loop control system with FOPI controller is stable, it is proposed to use the integral quality indices (IAE and ISE) and, respectively, equation (14) and inequality (18) to determine the range of $K_{P}$ and $K_{I}$ parameters. The system is stable in accordance with Bounded Input Bounded Output (BIBO) criterion, when the conditions are met:

1. The duration of simulation must be the same as the desired simulation time set at the process beginning.

2. Only successfully conducted simulations are considered: one needs to verify if the consecutive peaks of the output signal $y(t)$ are decreasing by measuring the difference between them. In affirmative case, the system is assumed to be stable.

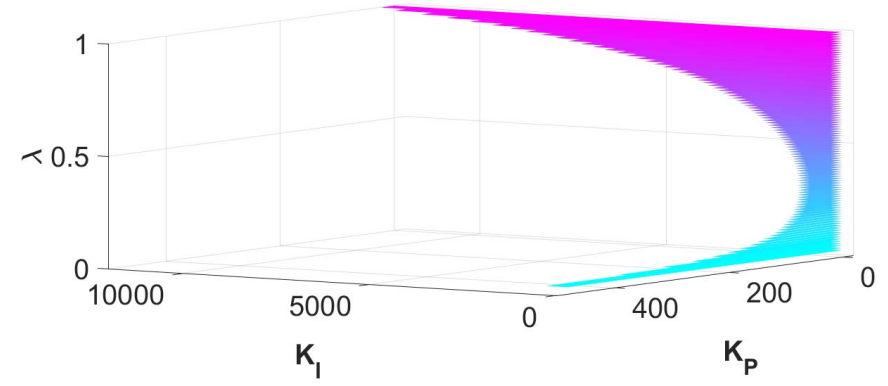

Fig. 2. Stability regions of parameters $K_{P}, K_{I}$ for change of $\lambda$ values from 0.01 to 1 for closed-loop control system with plant model (1): $\omega$ value from -200 to 200 (step: $4 \mathrm{rad} / \mathrm{s}$ ).

The search of controller sets from Fig. 2 in the designated space (for which the closed-loop control system is stable), to find the best tracking quality $\left(K_{P}, K_{I}\right.$ and $\lambda$ sets which ensure the possibly fast tracking and signal with minimal or no overshoot), may be based on recorded values of quality indicators (in this paper the IAE), or on another cost function, in accordance with selected optimization methods.

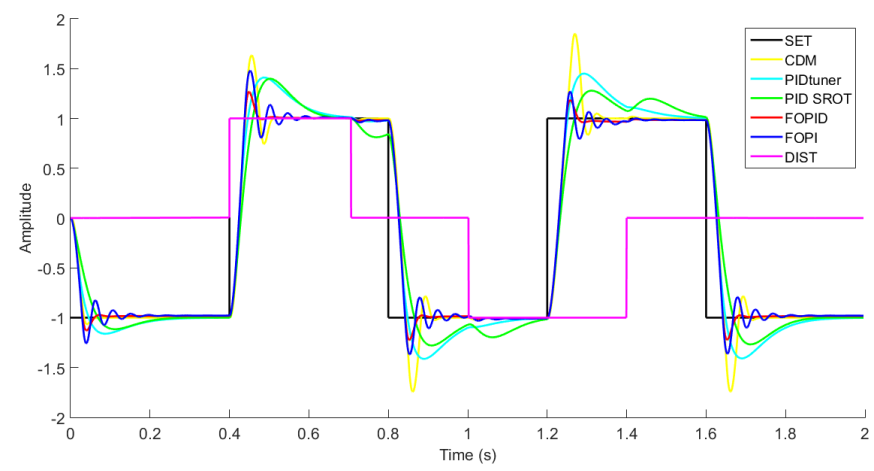

Fig. 3. Reference signal (SET) tracking in disturbed (DIST) systems (with CDM, PID, FOPID and FOPI controllers) at control signal constraints $\left(u_{\max }= \pm \emptyset\right)$. 


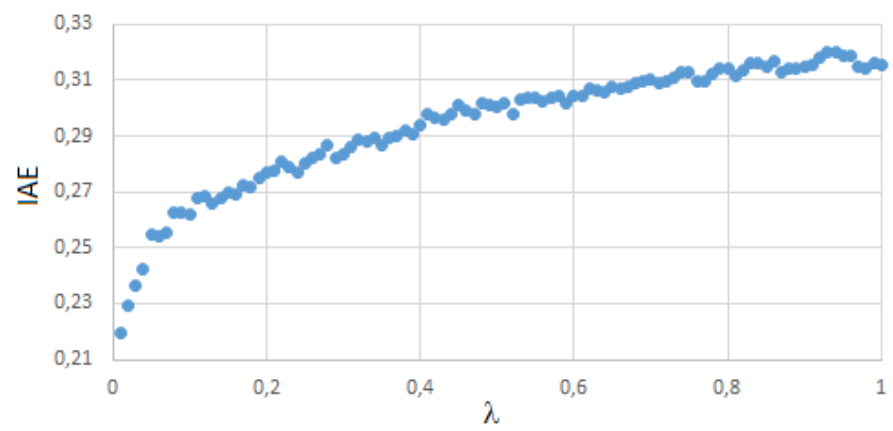

Fig. 4. IAE values as a function of $\lambda$ for FOPI controller type.

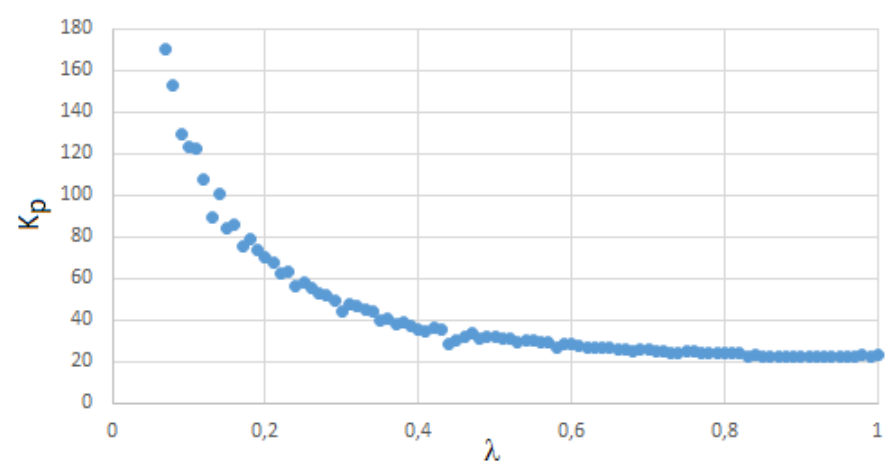

Fig. 5. $K_{P}$ values as a function of $\lambda$ for FOPI controller type.

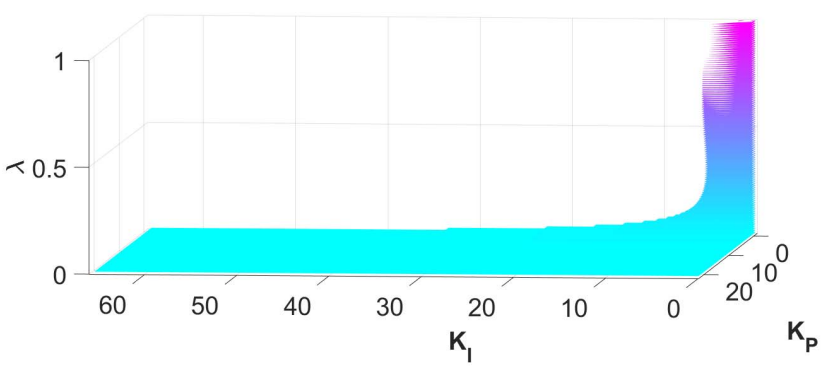

Fig. 6. Stability regions of parameters $K_{P}, K_{I}$ for change of $\lambda$ values from 0.01 to 1 for closed-loop control system with nominal plant model (1): $\omega$ value from $-\pi$ to $\pi$ (step: $2 \pi / 100$ ).

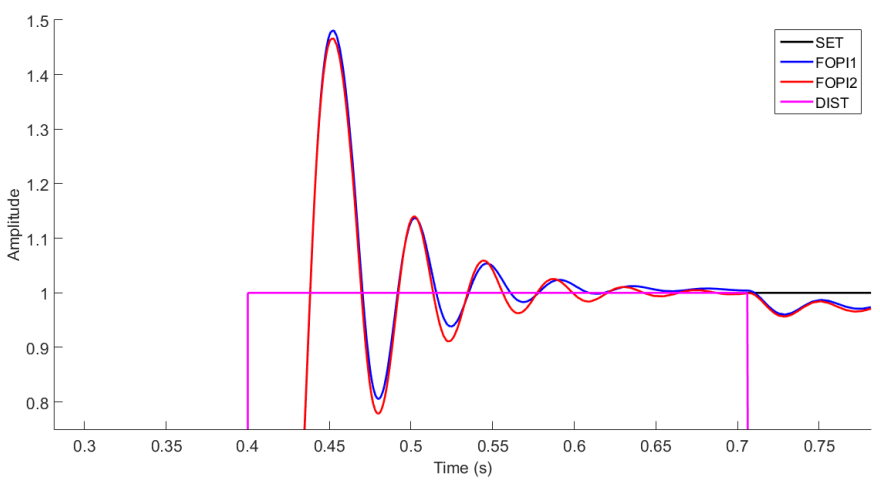

Fig. 7. Comparison of tracking effectiveness for two FOPI controllers: FOPI1 $\left(K_{P}=32.27, K_{I}=10.09, \lambda=0.49, I A E=0.30\right)$ and FOPI2 $\left(K_{P}=21.86, K_{\Gamma}=14.41\right.$, $\lambda=0.01, I A E=0.31)$.
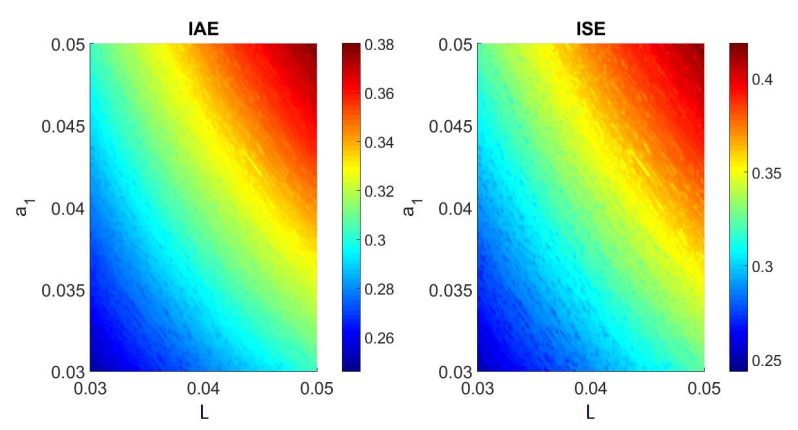

Fig. 8. IAE and ISE values due to the change of $a_{l}$ and $L$ propulsion unit model parameters.

\section{COMPARATIVE TESTS RESUlts}

In this paper, results are presented for the model (1) from [11], in which $b_{0}=1, a_{0}=1, a_{1}=0.04, L=0.35$.

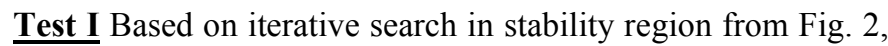
recorded tracking time courses and IAE values for the problem illustrated in Fig. 3, FOPI controller sets were selected ( $K_{P}=32.27, K_{I}=10.09, \lambda=0.49$ ), fulfilling the assumptions from the previous section. The FOPI controller effectiveness was compared with others from [11] and [14] as depicted in Fig. 3. The main aim was achieved: better tracking quality was obtained (in the presence of step type disturbances and control signal saturation) for the best tunes of PID (details in [14]) and PI type controllers. As a disadvantage one may regard appearing oscillations.

If the controller sets search relied solely on analysis of IAE values, one may conclude that simple $\mathrm{P}$ controller type is enough for tracking control purposes ( 83 from 100 best IAE values were obtained for $K_{I}=0$ and $\lambda$ from 0.01 to 1 ). However, time courses show that overshoot is not decreased to zero. In the remaining 17 variants, also the use of the integrator does not guarantee the elimination of steady-state error (it should be remembered that one must deal here with a fractional-order for integrator part of controller).

Based on Fig. 4 and Fig. 5, for a similar range of $K_{I}$ values, in lower range of $\lambda$, it is possible to enforce the improvement (decrease) of the IAE (by the increase of $K_{P}$ ). In practice, in the controller sets search, a useful range of values included: $K_{P}$ from 24 to $33, K_{I}$ from 10 to 40 and $\lambda$ from 0.49 to 0.79 .

Test II Relative to Test I, a range of $\omega$ values was changed from $-\pi$ to $\pi$ (step: $2 \pi / 100$ ) [25]. It was evaluated whether the decrease of $\omega$ value (and thereby space of sets for which closed-loop system is stable), will cause a radical deterioration in the reference signal tracking quality.

The best tracking performance and lowest value of IAE, were obtained for sets: $K_{P}=21.86, K_{I}=14.41$ and $\lambda=0.01$. Despite the reduction on the search space size for FOPI controller sets (see Fig. 6), there was no loss of tracking quality (see Fig. 7).

Test III The controller obtained in Test II was validated in a context of robustness on parametric uncertainty (see Fig. 8). Model parameters $a_{1}$ and $L$ were changed up to $25 \%$ 
of nominal values. The robustness is smaller than for nominal model, only for simultaneously bigger values of $a_{l}$ and $L$, that is, IAE and ISE values grow.

\section{CONCLUSION}

In this paper, the SCoMR-FOPI procedure for control system synthesis with the FOPI controller and model of UAV's propulsion unit is proposed, by using the Hermite-Biehler and Pontryagin theorems. This method provides the better tracking quality than classical PID and CDM controllers. However, its performance is less efficient than the fractional order PID control. The reduction of the controller parameters number from five in FOPID type controller $\left(K_{P}, K_{I}, K_{D}, \lambda, \mu\right)$ to three in FOPI, has it pros and cons. One may use HermiteBiehler and Pontryagin theorems to predefine a stability region for controller sets search. The possibility to use two more sets (degrees of freedom: $K_{D}, \mu$ ) on FOPID controller generates increased computational complexity. In this field, FOPI controller may be an alternative. In fact, it provides high robustness and may be used to control a propulsion unit model, which parameters vary from real plant parameters.

Further research will include discrete version of the proposed FOPI controller by Oustaloup approximation and optimization techniques to shorten the tuning time.

\section{REFERENCES}

[1] V.S. Akkinapalli, G.P. Falconi, and F. Holzapfel, "Attitude control of a multicopter using L1 augmented quaternion based backstepping", 2014 IEEE Inter. Conf. on Aerospace Electronics and Remote Sensing Technology (ICARES), pp. 170-178, Yogyakarta, Indonesia 2014.

[2] D. Aleksandrov and I. Penkov, "Optimization Mini Unmanned Helicopter Energy Consumption by Changing Geometrical Parameters of Coaxial Rotor Pairs", Topical Problems in the Field of Electrical and Power Engineering, online, pp. 139-141, 2012.

[3] V.M. Arellano-Quintana, E.A. Portilla-Flores, E.A. Merchan-Cruz, and P.A. Nino-Suarez, "Multirotor Design Optimization Using a Genetic Algorithm", In 2016 International Conference on Unmanned Aircraft Systems (ICUAS), pp. 1313-1318, Arlington, USA 2016.

[4] R. Bellman and K.L. Cooke, "Differential-Difference Equations", Academic Press, vol. 45, no. 6 DOI: 10.1002/zamm.19650450612, 1963.

[5] A. Bondyra, S. Gardecki, P. Gąsior, and W. Giernacki, "Performance of coaxial propulsion in design of multi-rotor UAVs", Advances in Intelligent Systems and Computing, Springer, pp. 523-531, 2016, DOI: $10.1007 / 978-3-319-29357-8 \_46$.

[6] A.S. Brandão, F.N. Martins, and H.B. Soneguetti, "A Vision-based Line Following Strategy for an Autonomous UAV", 2015 12th International Conference on Informatics in Control, Automation and Robotics (ICINCO), pp. 314-319, Colmar, France 2015.

[7] A.-C. Brezoescu, T. Espinoza, P. Castillo, and R. Lozano, “Adaptive Trajectory Following for a Fixed-Wing UAV in Presence of Crosswind", Journal of Intelligent and Robotics Systems, vol. 69, Issue 1-4, pp. 257-271, 2013.

[8] R. Caponetto, G. Dongola, L. Fortuna, and I. Petras, "Fractional Order Systems Modeling and Control Applications", World Scientific Series on Nonlinear Science: Singapore, Series A, no. 72, 2010.

[9] P. Castillo, R. Lozano, and A.E. Dzul, Modelling and Control of MiniFlying Machines, Springer, London, 2005.

[10] YQ. Chen, I. Petras, and D. Xue, "Fractional Order Control A Tutorial", American Control Conference, pp. 1397-1411, St. Louis, USA 2009.

[11] S. Gardecki, W. Giernacki, and J. Gośliński, "Speed Control of Drive Unit in Four-Rotor Flying Robot", In Proc. of the 2013 10th International Conference on Informatics in Control, Automation and Robotics (ICINCO), pp. 245-250, Reykjavik, Iceland 2013, DOI: $10.5220 / 0004477002450250$.

[12] P. Gąsior, A. Bondyra, S. Gardecki, W. Giernacki, and A. Kasiński, "Thrust estimation by fuzzy modeling of coaxial propulsion unit for multirotor UAVs", In Proceedings of the 2016 IEEE International Conference on Multisensor Fusion and Integration for Intelligent Systems (MFI), pp. 418-423, Baden-Baden, Germany 2016, DOI: 0.1109 /MFI.2016.7849524.

[13] W. Giernacki and J.P. Coelho, "Effective tuning approaches of PID fractional-order speed controller for multirotor UAV's motor-rotor".

[14] W. Giernacki, "Near to optimal design of $\mathrm{PI}^{\lambda} \mathrm{D}^{\mu}$ fractional-order speed controller (FOPID) for multirotor motor-rotor simplified model", In Proc. of the 2016 International Conference on Unmanned Aircraft Systems (ICUAS), pp. 320-326, Arlington, USA 2016, DOI: 10.1109/ICUAS.2016.7502516.

[15] W. Giernacki, D. Horla, T. Sadalla, and J.P. Coelho, "Robust CDM and Pole Placement PID Based Thrust Controllers for Multirotor MotorRotor Simplied Model", 2016 International Siberian Conference on Control and Communications (SIBCON), pp. 1-5, Moscow, Russia 2016, DOI: 10.1109/SIBCON.2016.7491826.

[16] W. Giernacki and T. Sadalla, "Comparison of tracking performance and robustness of the simplied model of multirotor aerial robot with CDM and PID (with anti-windup compensation) controllers", Journal of Control Engineering and Applied Informatics.

[17] S. Hafasi, K. Laabidi, and R. Farkh, "Synthesis of a fractional PI controller for a first-order time delay system", Trans. of the Institute of Measurement and Control, vol. 35, no. 8, pp. 997-1007, 2013.

[18] K. Mathe, L. Busoniu, L. Barabas, L. Miclea, J. Braband, and C. Iuga, "Vision-Based Control of a Quadrotor for an Object Inspection Scenario", In Proc. of the 2016 International Conference on Unmanned Aircraft Systems (ICUAS), pp. 849-857, Arlington, USA 2016.

[19] C.A. Monje, YQ. Chen, B.M. Vinagre, D. Xue, and V. Feliu, Fractionalorder Systems and Controls. Fundamentals and Applications, Springer, London, 2010.

[20] M. Muhlegg, P. Niermayer, G.P. Falconi, and F. Holzapfel, "L1 Fault Tolerant Adaptive Control of a Hexacopter with Control Degradation", In Proc. of the 2015 IEEE Conference on Control Applications (CCA), pp. 750-755, Sydney, Australia 2015, NSW.

[21] M. Nieuwenhuisen, D. Droeschel, M. Beul, and S. Behnke, "Autonomous Navigation for Micro Aerial Vehicles in Complex GNSSdenied Environments", Journal of Intelligent and Robotics Systems, pp. 1-18, 2015, DOI: 10.1007/s10846-015-0274-3.

[22] I. Petráš, "The fractional-order controllers: methods for their synthesis and application", Journal of Electrical Engineering, vol. 50, no. 9-10, pp. 284-288, 1999.

[23] I. Podlubny, "Fractional-order systems and $\mathrm{PI}^{\lambda} \mathrm{D}^{\mu}$-controllers", IEEE Transactions on Automatic Control, vol. 44, no. 1, pp. 208-214, 1999.

[24] T. Sadalla, D. Horla, W. Giernacki, and P. Kozierski, "Influence of time delay in fractional-order PI controller for a second-order oscillatory system with time-delay", in review.

[25] T. Sadalla, D. Horla, W. Giernacki, and P. Kozierski, "Stability Analysis and Tracking Performance of Fractional-Order PI Controller for a Second-Order Oscillatory System with Time-Delay”, 21th International Conference on Methods and Models in Automation and Robotics (MMAR), pp. 322-326, Międzyzdroje, Poland 2016, DOI: 10.1109/MMAR.2016.7575155.

[26] H. Saribas and S. Kahvecioglu, "Control of Quadrotor using Particle Swarm Optimization tuned Fractional Order PID Controller", $8^{\text {th }}$ Ankara Interantional Aerospace Conference, Ankara, Turkey 2015.

[27] J. Smith, C. Liu, and W.H. Chen, "Disturbance Observer Based Control for Gust Alleviation of a Small Fixed-Wing UAS”, In Proc. of the 2016 International Conference on Unmanned Aircraft Systems (ICUAS), pp. 97-106, Arlington, USA 2016.

[28] H. Won Kim and R.E. Brown, "A Comparison of Coaxial and Conventional Rotor Performance", Journal of the American Helicopter Society, vol. 55, 2009, DOI: 10.4050/JAHS.55.012004. 